\title{
ROOM-TEMPERATURE THZ IMAGING BASED ON ANTENNA-COUPLED MOSFET BOLOMETER
}

\author{
Thomas Morf ${ }^{1}$, Bernhard Klein ${ }^{1,3}$, Michel Despont ${ }^{1}$, Ute Drechsler ${ }^{1}$, Lukas Kull ${ }^{1}$, Dan Corcos ${ }^{2}$, \\ Danny Elad ${ }^{2}$, Noam Kaminski ${ }^{2}$, Matthias Braendli ${ }^{l}$, Christian Menolfi ${ }^{l}$, Marcel Kossel ${ }^{l}$, \\ Pier Andrea Francese ${ }^{1}$, Thomas Toifl, Dirk Plettemeier ${ }^{3}$ \\ ${ }^{1}$ IBM Research - Zurich, Switzerland, ${ }^{2}$ IBM Research - Haifa, Israel, \\ ${ }^{3}$ Communications Laboratory, Chair for RF Engineering, Technical University of Dresden, Germany
}

\begin{abstract}
We report on the design, fabrication and measurements of a new THz sensor concept based on an antennacoupled MOSFET bolometer for room-temperature passive $\mathrm{THz}$ imaging for security and medical-diagnostic applications. The device is fabricated in a $180-\mathrm{nm}$ CMOS SOI technology followed by a post-CMOS MEMS process. In this sensor, the antenna absorbing the $\mathrm{THz}$ electromagnetic field is directly coupled to the bolometer for maximum energy collection, whereas its design aims at minimizing its thermal mass as is necessary for fast frame rates. DC measurements before and after the MEMS process as well as thermal time constant and $\mathrm{THz}$ antenna measurements are presented.
\end{abstract}

\section{INTRODUCTION}

THz-based imaging in the frequency range of 0.5 to 1.5 THz has attracted great research attention for medical and security applications [1, 2]. Passive imaging is preferred as building $\mathrm{THz}$ sources is very challenging and no exposure of objects to RF fields is needed. However, objects at $300 \mathrm{~K}$ emit very little energy in the frequency range of interest. One has to expect about two to three orders of magnitude less power than in the IR range, where the signal peaks around $10 \mu \mathrm{m}$ wavelength. Current research focuses on cooled bolometers or bolometers using unconventional materials and concepts [2], including enhancing the sensitivity with thermal antennas $[1,3]$ and MOSFET-based mixers for detecting the radiation [4]. In our approach, see Fig. 1, the $\mathrm{THz}$ radiation received by a wide-bandwidth antenna is converted into heat by a termination resistor. Next to it is a MOSFET that acts as a temperature sensor thus indirectly detecting the $\mathrm{THz}$ radiation [3]. This approach is expected to enable room-temperature passive $\mathrm{THz}$ imaging with good sensitivity, wide bandwidth and adequate frame rate.

\section{DESIGN}

The design was done in a low-cost IBM 180-nm four-metal-layer SOI CMOS process (CSOI7RF). Four metal layers are sufficient for this application. The process has a $1 \mu \mathrm{m}$ tick buried oxide (BOX) layer, which is used as an etch stop and provides ample etch margin for the releasing process.

The RF design was done in HFSS from Ansoft (Ansys), a 3-D full-wave electromagnetic field simulator based on the finite element method. The pixel is basically an antenna- coupled bolometer. A wideband antenna is needed for collecting a sufficient amount of $\mathrm{THz}$ radiation.

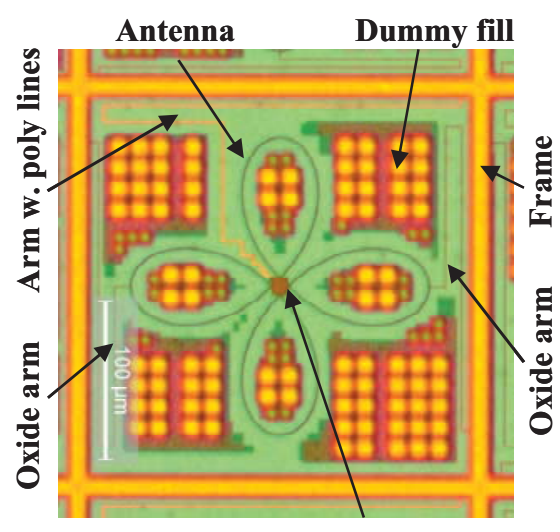

Platform, term. resistor \& sensor

Figure 1: Chip photograph before the post CMOS process of a single $\mathrm{THz}$ receiving pixel, showing the antenna, the sensor, and additional metals used as mask during the MEMS process.

The antenna should have an effective antenna area, i.e. the area from which it receives the signal, of about the size of the pixel. Moreover, it should cover the frequency band of interest, which with $1 \frac{1}{2}$ octave is already wideband. The antenna design is also limited to planar antennas; a metallic planar reflector, formed by the package as shown in Fig. 2

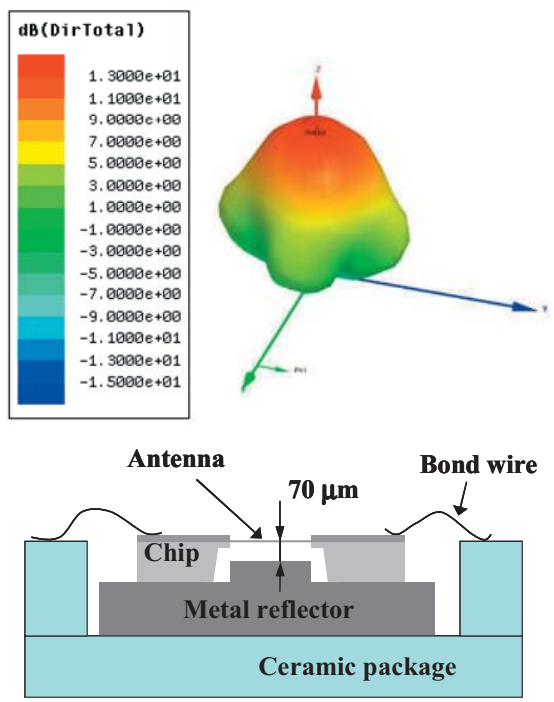

Figure 2: Simulated antenna radiation pattern at $1.4 \mathrm{THz}$ (after post-CMOS process) of the antenna shown in Fig. 1 including back-side reflector (package) and pixel frame. 
increases the antenna gain. The pixel's frame, which is made of metal 3, covers the wiring and acts as additional reflector for the antenna, increasing its antenna gain.

As the application is imaging, it is important that the antenna receives $x$ and $y$ polarizations about equally well. One other specification is the thermal mass of the antenna: The antenna is physically connected to the sensor, therefore a large metal area will increase the thermal mass of the sensor dramatically, making its response time very slow. Unfortunately, wideband antennas often are logarithmic antennas with large thermal mass. A novel cloverleaf antenna that offers an adequate bandwidth and a small thermal mass was developed. The antenna has two wings so that it receives both polarizations, see Fig. 1.

Next, the THz energy is converted into heat in matched termination resistors on a thermally insulated platform. In the vicinity of these resistors, there is a MOSFET that acts as temperature sensor. To see any temperature difference caused by the tiny $\mathrm{THz}$ signal expected, the thermal insulation of the sensor platform needs to be extremely high. The goal is a thermal resistance of approx. $10^{8} \mathrm{~K} / \mathrm{W}$. This can be achieved with very narrow and long holding arms. However, this requirement leads to a very fragile and soft structure requiring special attention in the design and the MEMS releasing process.

Because of the narrow metal structures, built from metal 1, the antenna has a small thermal mass, but also a rather high RF loss. However, as the antenna is physically connected to the sensor, the RF loss in the antenna arms will heat up the antenna and by heat conduction also the sensor, but, in contrast to conventional antennas, here this signal is not lost. In Fig. 2 a simulated radiation pattern of this antenna with reflector at $1.4 \mathrm{THz}$ is shown.

Moreover, in such ultrasoft free-standing devices, stress in the different film layers is critical. Hence, to keep the structure planar, supporting arms made out of low-thermal-conductivity silicon-oxide are used. Typically, the pixels fabricated consist of a $200-\mu$ m-diameter antenna coupled to a $10-\mu \mathrm{m} \times 10-\mu \mathrm{m}$ platform with the integrated MOSFET thermal sensor and supported by two $315-\mu \mathrm{m}$-long and 500-nm-wide oxide arms. The pixel is electrically connected by a two-wire $440 \mu \mathrm{m}$ long holding arm, containing a conductor formed of silicided polysilicon (gate material) and a conductor formed of silicided active silicon. The two conductors are surrounded by a protective oxide. Conductor widths are given by the minimum design rules of approx. $200 \mathrm{~nm}$.

\section{POST-CMOS PROCESSING}

A post-CMOS processing step follows the standard CMOS fabrication process. The antenna and the sensor are fabricated up to metal 1. The top most metal drawn, serve as masks for the MEMS process to avoid critical mask-alignment steps.

Figure 3 describes the post-CMOS process. After the standard CMOS process (Fig. 3a), the front-side oxide layers, including the $\mathrm{BOX}$, are etched by $\mathrm{CHF}_{3} \mathrm{RIE}$ using the top metal layers as built-in masks and a photo-resist mask (PR) to protect the wire bond pads. The BOX silicon substrate interface acts as an etch stop (Fig. 3b). Next, the mask metal is removed by means of wet etching (Fig. 3c). The all-dry releasing process consists of back-side etching of a cavity using DRIE; here again a PR mask defines the size and location of the cavity. This process is stopped a few tens of microns before the release of the MEMS structure (Fig 3d). The release is achieved by an isotropic $\mathrm{SF}_{6}$ RIE step for the final tens of microns from the front side (Fig. 3e). Note that the fill structure shown in Fig. 1 is required by the foundry processing rules. A metal density in a defined range is required to enable chemical mechanical polishing (CMP). For the chip layout and the MEMS process, these fill structures are undesirable. Custom fill structures are used rather than the a)

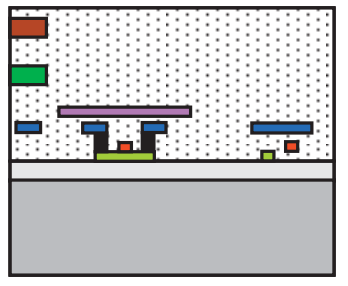

d)

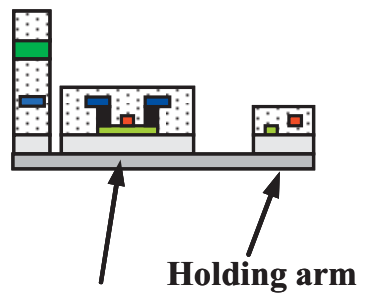

Platform b)

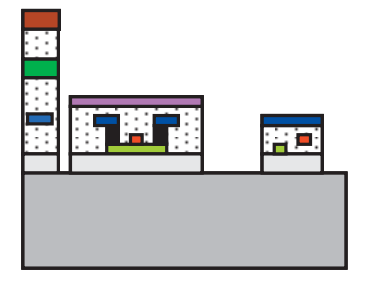

e)

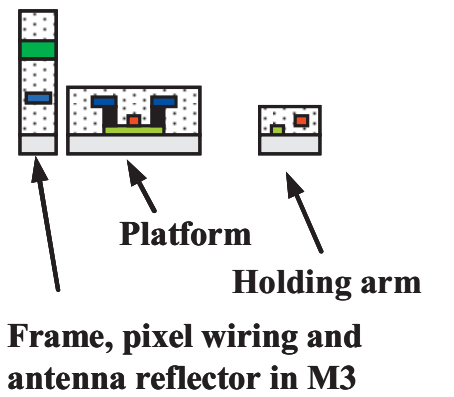
antenna reflector in $\mathrm{M3}$ c)

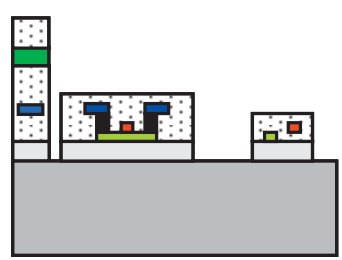

$\square$ Active Silicon

$\square$ Poly

$\square \quad$ Metal 1

$\square \quad$ Metal 2

$\square \quad$ Metal 3

$\square$ Metal 4

$\because \cdot$ Oxide

$\square$ Buried oxide

$\square$ Wafer

Figure 3: MEMS process. a) Cross section after CMOS process. b) Front-side oxide etch. c) Metal mask etch. d) Back-side Si etching. e) Release front-side etch. 


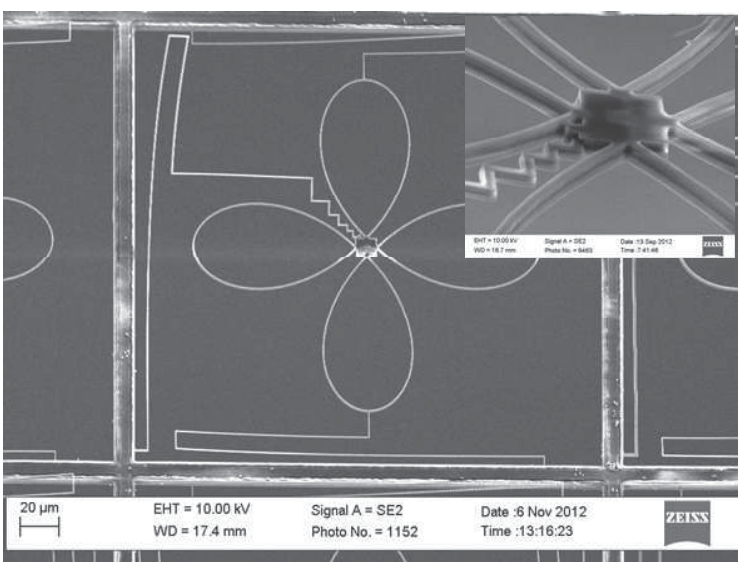

Figure 4: SEM picture of a single pixel after the postCMOS processing step. Pixel size is $250 \mu \mathrm{m}$ by $250 \mu \mathrm{m}$. The frame around the pixel acts as reflector for the antenna and provides mechanical support.

automatically generated small fill structures because these larger custom fill structures have fewer tendencies to stick and are easier to remove during the MEMS process.

Figure 4 shows SEM pictures of a completely released pixel. The cloverleaf antenna with three holding arms is clearly visible. The insert shows the platform in the center of the antenna. Practically all fill structures have been removed successfully.

\section{MEASURED RESULTS}

Current vs. voltage measurements before and after the release process are shown in Fig. 5, and reveal a slight shift in the FET threshold voltage that is attributed to a difference of the stress in the device. The PN diode characteristic visible in reverse direction is due to the fact that the NMOS FET contains a local body contact shortening source to body. The variation between chips observed after the release process is small.

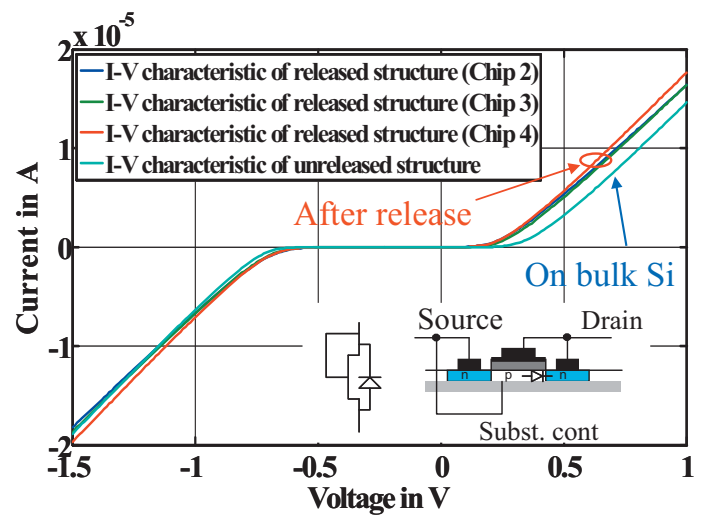

Figure 5: Current vs. voltage measurements of a single pixel before (dashed light-blue line) and three devices on three different chips after the MEMS process. The local body contact forms a parasitic PN diode.

Figure 6 shows the thermal time constant in vacuum (at $10^{-2}$ mbar) of the pixel shown in Figs. 1 and 4. First a negative pulse is applied to heat up the device, and next the diode's current is recorded vs. time for a small bias, which causes negligible self-heating. Here the diode acts as temperature sensor. The measured time constant, which corresponds to the 1/e amplitude of the pulse, is $53 \mathrm{~ms}$; this value is in reasonable agreement with the simulation results.

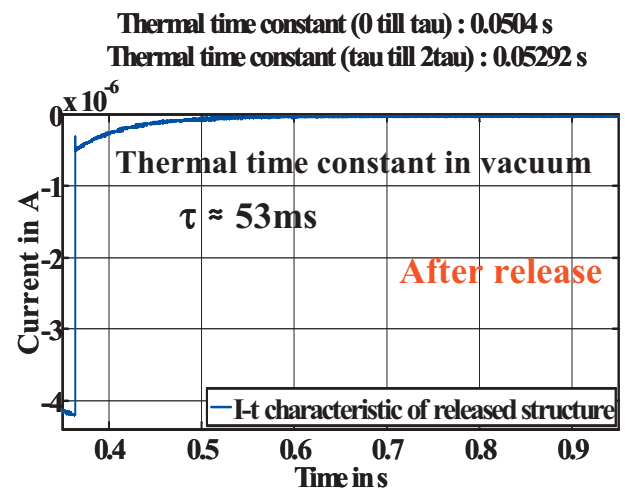

Figure 6: Measured thermal time constant of pixel shown in Fig 4 in vacuum at $10^{-2}$ mbar. First a negative pulse is applied to heat up the device, and then the diode current is recorded vs. time for a small bias, which causes negligible self-heating. (FET acts as temperature sensor).

$\mathrm{THz}$ measurements have been performed in which the sensor was operated in air and irradiated by a linearly polarized chopped $655 \mathrm{GHz}$ signal emitted from a horn antenna. The sensor was rotated along two axes, and the sensor current was recorded by a lock-in amplifier as a function of the angles. Figure 7 shows the antenna's receiving pattern normalized to an antenna gain of $0 \mathrm{~dB}$. As shown in Fig. 2, a reflector formed by the package was

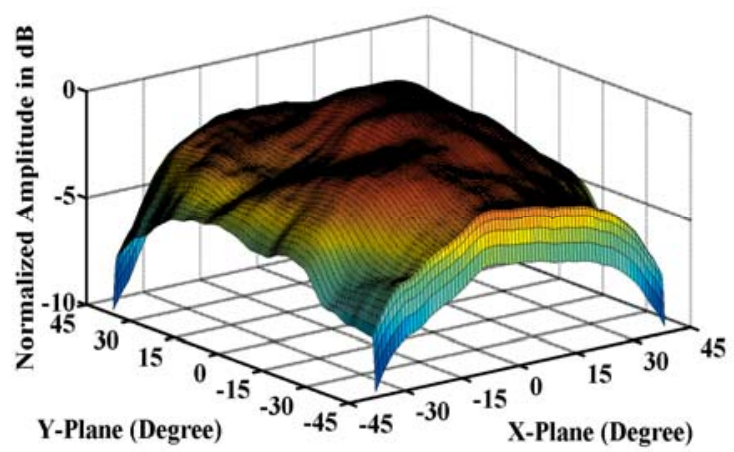

Figure 7: Measured antenna radiation pattern of the antenna shown in Figs. 1, 2 and 4, measured at $655 \mathrm{GHz}$. The gain is normalized to $0 \mathrm{~dB}$.

used for increasing the gain. Measurements in air result in a loss of more than two orders of magnitude in responsivity, compared to the values in vacuum; this is due to the thermal conductivity of air. On the other hand, antenna pattern measurements in vacuum are problematic owing to the influence of the vacuum enclosure (reflection, shadowing) on the antenna pattern. The antenna pattern shown in Fig. 7 is in reasonable agreement with the HFSS simulations. 


\section{CONCLUSION}

The design and measurement results of a novel $\mathrm{THz}$ detector for imaging in the 0.5 to $1.5 \mathrm{THz}$ frequency band were described. The antenna-coupled bolometer was designed and optimized using 3D field simulations. After a standard SOI CMOS fabrication process, a MEMS post-process was performed to fully release the MEMS structure. The remaining structure is a narrow antenna with low thermal mass and holding arms with low thermal conductivity. This enables the very high thermal insulation needed to receive low-level $\mathrm{THz}$ signals. The MEMS process is quite challenging because of the fragile structures. On the other hand, once released, the structure is robust owing to its very small mass. DC thermal and $\mathrm{THz}$ measurements proved that the sensor survived the MEMS process and is fully functional. Based on measurements in air, we validated our models of the sensor's electro-magnetic and thermal responsivity. Vacuum conditions will be necessary for achieving the high sensitivity needed in passive imaging applications.

\section{REFERENCES}

[1] L. Gitelman, S. Stolyarova, S. Bar-Lev, Z. Gutman, Y. Ochana, and Yael Nemirovsky, "CMOS-SOI-MEMS Transistor for Uncooled IR Imaging, IEEE Trans. Electron Devices, vol. 56, no. 9, pp. 1935-1942, September 2009.

[2] Y. Lee, Principle of Terahertz Science and Technology, Springer Science+Business Media, LLC, New York, NY, 2009.
[3] D. Corcos, I. Brouk, M. Malits, A. Svetlitza, S. Stolyarova, A. Abramovich, E. Farbe, N. Bachar, D. Elad and Y. Nemirovsky, "The TeraMOS Sensor for Monolithic Passive THz Imagers", Proc. 2011 Int'l. Conf. on Microwaves, Communications, Antennas and Electronics Systems (COMCAS), Tel Aviv, Israel, November 7-9, 2011.

[4] H. Sherry, J. Grzyb, Y. Zhao, R. Al Hadi, A. Cathelin, A. Kaiser and U. Pfeiffer, "A 1k Pixel CMOS Camera Chip for 25 fps Real-Time Terahertz Imaging Applications", Proc. 2012, IEEE Int'l Solid- State Circuits Conf. (ISSCC 2012), San Francisco. CA, February 19-23, 2012, pp. 252-253.

\section{ACKNOWLEDGEMENT}

We would like to thank Prof. Dr. Ulrich Pfeifer, Richard Al Hadi and Hans Keller from the University of Wuppertal for their very valuable help with the $\mathrm{THz}$ measurements.

\section{CONTACT}

Thomas Morf, IBM Research - Zurich, Säumerstrasse 4, 8803 Rüschlikon, Switzerland Tel: +41-44 724 8515; Fax:+41-44-724 8955; E-mail: tmr@zurich.ibm.com

The research leading to these results has received funding from the European Union Seventh Framework Program (FP7/2007-2013) under grant agreement no. [288442] 